Vol.01/ No. 01

Pages: 24-32

http://irojournals.com/jscp/

DOI: https://doi.org/10.36548/jscp.2019.1.003

\title{
STUDY ON HERMITIAN GRAPH WAVELETS IN FEATURE DETECTION
}

\author{
Dr. Samuel Manoharan, \\ Professor, Department of Electronics, \\ Bharathiyar College of Engineering and Technology, \\ Thiruvettakudy, Karaikal, India. \\ Email id: jsamuel@bcetedu.in
}

\begin{abstract}
The enormous information flow in our day today life, initiates the necessitates of the identifying the valuable data that are to be concentrated. In case of image segmentation and signal processing, the feature detection takes up the role of fixating to the data that are to be focused. Thus directing to the pixels or information that are to be concentrated eliminating the time and the energy wastage in examining the pixels or the information's that are of least important. The paper is the study, focusing on the advantages of utilizing the Hermitian wavelet transform incorporated with the graph wavelet in the feature detection, leading to an accurate identification of the information to be processed further.
\end{abstract}

Keywords: Wavelet transform, Hermitian wavelet, Graph wavelet, Feature detection, Advantages, Signal processing, Image processing

\section{Introduction}

The human brain are capable of processing enormous of information's such as images and videos, the eye shows interest in an object and the brain immediately arises, by extending help in identifying the attributes of the object related to its texture, shade and the shape to match the object, the same concept of the human brain working in identifying the object is followed in the area of the computer vision too. The process that involves the steps in deciding with what to focus on is called as the feature detection in the computer vision. Kenneth $\mathrm{R}$ castle man, defines the features as "one or more measurement of some quantifiable property of an object, processed so that it quantifies some of the important attributes of the objects [2], alternatively the feature could be defined as the more interesting section of an image. In image processing the feature detection helps in accurately estimating the pixels to be focused to elude the unnecessary wastage on time and energy in identifying the part that are of no significance. 
Journal of Soft Computing Paradigm (JSCP) (2019)

Vol.01/ No. 01

Pages: 24-32

http://irojournals.com/jscp/

DOI: https://doi.org/10.36548/jscp.2019.1.003

Some of the basic type of features are, blobs, lines, circles, corners. The robust feature detectors enables to identify the features in a more reliable way among innumerable images. Based on the detection criteria it can be determined whether a feature could be identified under the picture with the position invariance, scale invariance and rotation invariance. The feature detection incorporates the methods of the computing concepts to arrive at a local decision for every point to come up with the result that has identified whether there is a feature of given type or not, the resulting features detected would be the subparts of the original object, most probably the feature detectors are engaged with localization, mapping in the landscape, disease diagnoses, finger print detection, face detection, etc. employing computer algorithms that are equipped with the feature detectors the table.1 below gives the details of the types of the feature detectors available and their essential classifications

The feature detection ensure the unwanted wastage of time of energy by deciding with the feature to be concentrated, so in any computer vision process the feature detection takes the initial stage for further proceeding with the extraction and the description. The feature detection becomes extremely essential in the image processing or the signal processing areas where the originality of the information is hidden behind many unwanted information's

The feature detection could be otherwise meant as retrieval of the valuable information from the cluster of information's that holds both valuable and the invaluable information's engaging the feature detectors would enable the saving a considerable amount of time and energy.

The table.1 elaborating the few basic feature detectors, form the survey gathered it was understood that the graph wavelets could also be engaged in the feature detection. So the paper is to study the feature detection using the graph wavelets and the importance of engaging the Hermitian graph wavelet in the feature detection.

The remaining paper is organized with the 2 . The feature detection using the wavelets, 3 . The significance of the graph wavelets, 4. Importance of the Hermitian graph wavelet transform in the feature detection 5. Conclusion.

\section{Wavelets in the Feature Detection}

ISSN: 2582-2640 (online) 
Journal of Soft Computing Paradigm (JSCP) (2019)

Vol.01/ No. 01

Pages: 24-32

http://irojournals.com/jscp/

DOI: https://doi.org/10.36548/jscp.2019.1.003

The wavelets are similar to signals or oscillated waves that move ahead in an increasing rate from its initial position and tread back to its initial position from the forward position. These wavelets were purposely designed with the distinct property that make them applicable in the field of signal processing. The wavelets are incorporated with the damaged signals to pull out the valuable information's or even simply employed with the signals obtained to extract features for the further analysis, usually the wavelets are engaged in the image as well as audio, the video and any other data processing for the extraction of the valuable information's to further proceed with the analysis for further improvement or detection or classification.

\begin{tabular}{|c|c|c|c|}
\hline Feature detector & Edge Detector & Corner detector & Blob Detector \\
\hline Canny & $\checkmark$ & & \\
\hline FAST & & & \\
\hline PCBR & & & \\
\hline Harris \&Stephen & $\checkmark$ & & \\
\hline Shi \& Thomas & & $\checkmark$ & \\
\hline Susan & $\checkmark$ & & \\
\hline Level curve Curvature & & & \\
\hline Sobel & $\checkmark$ & & \\
\hline Laplacian of Gaussian & & & \\
\hline Difference of Gaussian & & & \\
\hline MSER & & & \\
\hline Kayyali & $\checkmark$ & & \\
\hline Grey-level Blobs & & & \\
\hline Determinant Hessian & & & \\
\hline
\end{tabular}

Table .1 Basic Feature Detectors

The wavelet transforms can be widely categorized into three major classes as the continuous, discrete and multiresolution based. The continuous wavelet is an explicit tool with the more complete representation of the object. Some of the application that utilize the wavelet transform are themedical field, utilizing he wavelet entropy in the preclinical diagnosis for the classification of the MRI [9], in the computed tomography scan [10] in ultrasound [12] 
Journal of Soft Computing Paradigm (JSCP) (2019)

Vol.01/ No. 01

Pages: 24-32

http://irojournals.com/jscp/

DOI: https://doi.org/10.36548/jscp.2019.1.003

etc. they are also employed in many other fields such as face detection and finger print detection and land scape etc. But the less efficiency and are computationally intensive in handling the large set of data that are unlabeled so this brings in the necessity of the graph wavelets.

\section{Significance of the Graph Wavelet}

The latest developments in the signal processing has lengthened the conventional methods of the wavelet transform to the domains that are with irregularities, that is the graph. The functions of the graph being almost similar to the operations of the conventional wavelet transforms differs on the basis of detecting as the wavelet transforms detect the transients and the graph wavelets are useful in detecting the discontinuities of the functions that are defined over the graph [14] the graph wavelet transforms the images to the graph domain and identifies the discontinuities in the pixel domain, the graph wavelets were prominent in the field of the image segmentation, [15],[16],[17],[18]. the graph wavelet methods in the image segmentation considers the intensity values or the edge values as the function that are defined on the graph, the graphs of equal sizes would be generated for the equal sized images, with the changes being reflected on the graph function. The steps below show the framing of the graph wavelet.

The two operations that are used in the wavelet transform are dilation and translation that could be easily meant as the scaling and the localization. This process of the wavelets are defined over the vertices of the weighted graph, that serves as the flexible option for the structuring the large number of the important applications [8] is the graph wavelet

The graph wavelet are formed by the decomposition of the Laplacean matrix $M * M$, where $M$ is the vertices of the graph, for the graph $G=(V, E)$ weighted undirected graph with the Laplacean $L=D-A$, the wavelets are defined with the scaling achieved [13] as shown in the equation (1)

$$
T_{g}^{t}=g(t L)
$$

And the localization characterizing the $\varphi_{t, j}=T_{g}^{t} \Delta J$ as a result of the delta at vertex $\mathrm{j}$ so the graph wavelet coefficient of $x$ is given as in equation (2)

ISSN: 2582-2640 (online) 
Journal of Soft Computing Paradigm (JSCP) (2019)

Vol.01/ No. 01

Pages: 24-32

http://irojournals.com/jscp/

DOI: https://doi.org/10.36548/jscp.2019.1.003

$$
W_{x}(t, j)=T_{g}^{t} x(J)=\sum_{n=0}^{M-1} g\left(t \omega_{n}\right) x(n)^{\prime} \pi_{n}(J)
$$

Since the graph wavelet converts the original images to graph domain make the tasks of the feature detection easy enabling the identification of appropriate pixels that are necessary from the object eluding the unnecessary features that cause enormous energy and time wastage. The next section is to explain the Hermitian graph wavelet (HGW) in feature detection. Before moving on with the efficiency and the necessity of the HGW, let us move to the explanation that details the Hermitian wavelet.

\section{Hermitian wavelet Transform}

The Hermitian wavelets are well suited in detecting the singularity in the signals. The signal singularity holds the valuable information's that are to be processed from the innumerable information's that were gathered from an application. From the available various family of wavelets, such the Gaussian and Mexican hat, the Hermitian wavelet belongs to the family of the continuous wavelet, the Hermitian wavelets includes the family of the Gaussian wavelet and the Mexican hat wavelet as a particular case [25], the $n^{\text {th }}$ derivative of the Hermitian wavelet is the $n^{\text {th }}$ derivative of the Gaussian distribution, the equation (3) gives the Hermitian wavelet obtained from the Gaussian distribution. [21]

$$
\varphi_{n}(t)=(2 n)^{-\frac{n}{2}} c_{n} H_{e_{n}}(t) e^{-\frac{1}{2 n} t^{2}}
$$

The hermite polynomial is denoted by the $H_{e_{n}}(t)$ and $c_{n}$ is the normalization co-efficient, the pre-factor representing the resolution of the continuous wavelet transform for the Hermitian wavelet is given by the equation (4)

$$
C W T_{\varphi}=\frac{4 \pi n}{2 n-1}
$$

Where the $C W T_{\varphi}$ is the pre-factor in the resolution of the identity, the equation (4) shows that the Hermitian wavelets are permissible for all positive $n$. the following shows the examples of the Hermitian wavelets as the derivative of the Gaussian function with $\mu=0, \sigma=1, f(t)=\pi^{-\frac{1}{4}} e^{\left(-\frac{t^{2}}{2}\right)}$ the initial three derivatives are read as

$$
\begin{aligned}
f^{\prime}(t) & =-\pi^{-1 / 4} t e^{\left(-t^{2} / 2\right)} \\
f^{\prime \prime}(t) & =\pi^{-1 / 4}\left(t^{2}-1\right) e^{\left(-t^{2} / 2\right)} \\
f^{(3)}(t) & =\pi^{-1 / 4}\left(3 t-t^{3}\right) e^{\left(-t^{2} / 2\right)}
\end{aligned}
$$


Journal of Soft Computing Paradigm (JSCP) (2019)

Vol.01/ No. 01

Pages: 24-32

http://irojournals.com/jscp/

DOI: https://doi.org/10.36548/jscp.2019.1.003

The following gives the characteristics of the Hermitian wavelet by applying the Fourier transform for it. [22] The derived wavelet for the Hermitian applying the Fourier transform is given below (6)

$$
\pi(\omega)=\left(\omega^{2}+\omega\right) e^{-\frac{\omega^{2}}{2}}
$$

Where the Hermitian holds both the imaginary and the real part, the real part represents the Mexican hat wavelet that takes an oscillation of one and a half and the imaginary is the odd function and takes the oscillation of one, thus resulting in single oscillation taking a very low frequency band. The Hermitian mother wavelet can be extended to its daughter wavelet in terms of the shift parameter and the scale parameter.

\subsection{Hermitian Graph Wavelet Transform}

The survey on the Hermitian wavelet transform and the spectral graph wavelet ensures to provide with an automatic detection of the accurate features in the image and the signal processing, the wavelets are the analysis tools that provide with the better performance than the Fourier transform[26]. Hermitian wavelet transform incorporated with the spectral graph analysis, framing the Hermitian graph wavelet, characterize the signal in an improved manner, and enables to obtain the optimal features. The capability of the Hermitian wavelet in identifying the singularity characteristics [20], combined with the spectral graph wavelets that work on the data converted into a graphical domain, enhance the measures of locating the optimal features in the feature detection. As the signal singularity is contained with the significant features from the enormous data gathered, the Hermitian graph wavelet that paves way for the singularity detection the it has a great impact on the signal processing, fault diagnosing and the fault tolerance [22] the study also ensures that the Hermitian graph wavelets are very reliable, as they are less prone to the noise effects. The Hermitian wavelets are comprised of lower frequency bandwidth and very few number of grid points in addition the Hermitian graph wavelets can restrain the instantaneous phase of the signals as it holds the Fourier spectrum that tis real and does not cause any impact on the signal phase in the complex domain [24] the Hermitian graph wavelet shows a highest regularity in pointing out the singular point in the simulative signal. So the Hermitian graph wavelet poses the suitability in detecting the singularity characteristics of the signal than the Morlet wavelet the fig. 1 showing the time domain wave form of the cosine simulative signal[20] shows the superiority of

ISSN: 2582-2640 (online) 
Journal of Soft Computing Paradigm (JSCP) (2019)

Vol.01/ No. 01

Pages: 24-32

http://irojournals.com/jscp/

DOI: https://doi.org/10.36548/jscp.2019.1.003

the Hermitian compared to the Morlet wavelet obtaining an optimal result with the reduced dimension features containing most valuable information's.

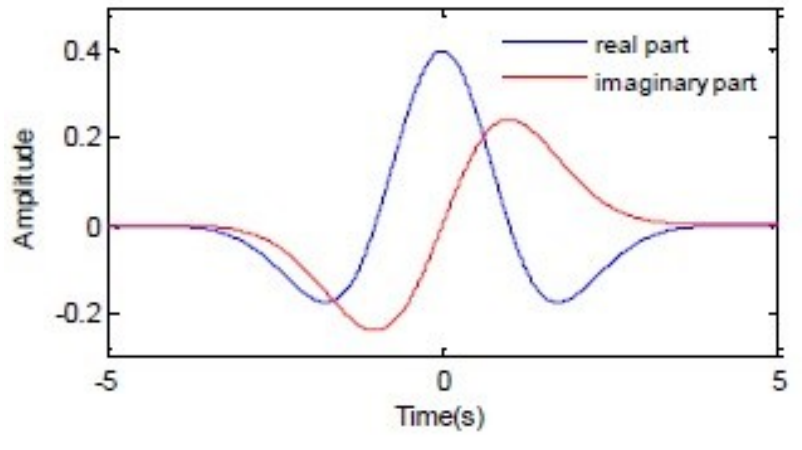

Fig. 1(a) Hermitian wavelet [20]

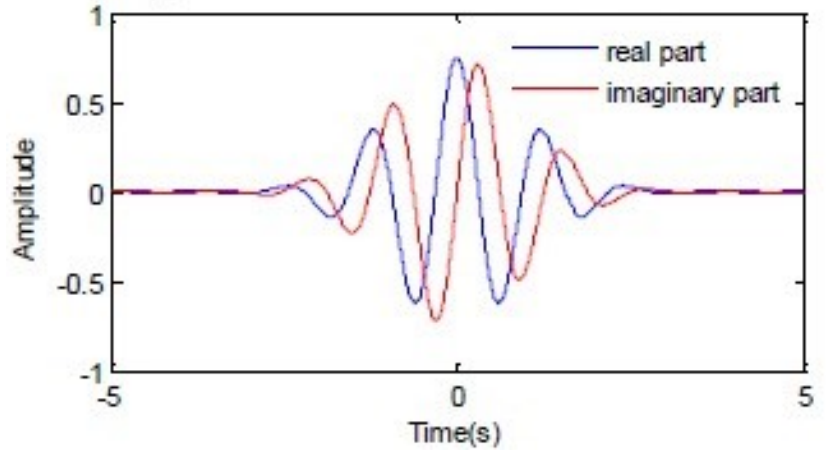

Fig .1(b) Morlets wavelet [20]

\section{Conclusion}

The study pursued in the paper to know the significance of the Hermitian graph wavelet in the feature detections, details the capability of the wavelet transform and the significance of the graph wavelet that focuses on the discontinuities in the information gathered, rather than noting the transients in the signals as such in wavelet transform. Based on the study the literatures reviewed the Hermitian graph wavelets shows much improved performed in the feature detection due to its capability of the singularity detection by the Hermitian and the ability of identifying the discontinuities in the object in the graph domain by the graph wavelets, thus enabling to focus on the accurate feature, enhancing the performance of the feature detection. Further the paper is proceed with the Hermitian graph wavelet in feature detection in the disease diagnoses as the future work.

\section{References}


Journal of Soft Computing Paradigm (JSCP) (2019)

Vol.01/ No. 01

Pages: 24-32

http://irojournals.com/jscp/

DOI: https://doi.org/10.36548/jscp.2019.1.003

[1] Brackx, Fred, Hennie De Schepper, and Frank Sommen. "A Hermitian setting for wavelet analysis: the basics." In Proceedings of the 4th International Conference on Wavelet Analysis and its Applications, University of Macau, China. 2005.

[2] Kenneth, R. "Castleman Digital Image Processing, 1996 Prentice-Hall." Chapter 14 (1979): 313-346.

[3] Li, Yali, Shengjin Wang, Qi Tian, and Xiaoqing Ding. "A survey of recent advances in visual feature detection." Neurocomputing 149 (2015): 736-751.

[4] Li, Shimiao. "A review of feature detection and match algorithms for localization and mapping." In IOP Conference Series: Materials Science and Engineering, vol. 231, no. 1, p. 012003. IOP Publishing, 2017.

[5] Lowe, David G. "Distinctive image features from scale-invariant keypoints." International journal of computer vision60, no. 2 (2004): 91-110.

[6] Harris, Christopher G., and Mike Stephens. "A combined corner and edge detector." In Alvey vision conference, vol. 15 , no. 50, pp. 10-5244. 1988.

[7] Rosten, Edward, and Tom Drummond. "Fusing points and lines for high performance tracking." In $I C C V$, vol. 2, pp. 1508-1515. 2005.

[8] Hammond, David K., Pierre Vandergheynst, and Rémi Gribonval. "The Spectral Graph Wavelet Transform: Fundamental Theory and Fast Computation." In Vertex-Frequency Analysis of Graph Signals, pp. 141-175. Springer, Cham, 2019.

[9] Zhou, Xing-Xing, Yudong Zhang, Genlin Ji, Jiquan Yang, Zhengchao Dong, Shuihua Wang, Guangshuai Zhang, and Preetha Phillips. "Detection of abnormal MR brains based on wavelet entropy and feature selection." IEEJ Transactions on Electrical and Electronic Engineering 11, no. 3 (2016): $364-373$.

[10] Arulmurugan, R., and H. Anandakumar. "Early detection of lung cancer using wavelet feature descriptor and feed forward back propagation neural networks classifier." In Computational Vision and Bio Inspired Computing, pp. 103-110. Springer, Cham, 2018.

[11] Silva, Sergio, Pyramo Costa, Maury Gouvea, Alcyr Lacerda, Franciele Alves, and Daniel Leite. "High impedance fault detection in power distribution systems using wavelet transform and evolving neural network." Electric Power Systems Research 154 (2018): 474-483.

[12] Acharya, U. Rajendra, G. Swapna, Savita Gupta, S. Vinitha Sree, Filippo Molinari, R. Garberoglio, Agnieszka Witkowska, and Jasjit S. Suri9 Sr. "Effect of Complex Wavelet Transform Filter on Thyroid Tumor Classification in 3D Ultrasound." (2019).

[13] https://www.macalester.edu/ dshuman1/Talks/Vandergheynst_Shuman_Marseille_11_17_2011.pdf

[14] Ozdemir, Alp, and Selin Aviyente. "Graph wavelet transform: Application to image segmentation." In 2014 48th Asilomar Conference on Signals, Systems and Computers, pp. 496-499. IEEE, 2014.

ISSN. 2582-2640 (online) 
Journal of Soft Computing Paradigm (JSCP) (2019)

Vol.01/ No. 01

Pages: 24-32

http://irojournals.com/jscp/

DOI: https://doi.org/10.36548/jscp.2019.1.003

[15] Shi, Jianbo, and Jitendra Malik. "Normalized cuts and image segmentation." Departmental Papers (CIS) (2000): 107.

[16]Felzenszwalb, Pedro F., and Daniel P. Huttenlocher. "Efficient graph-based image segmentation." International journal of computer vision 59, no. 2 (2004): 167-181.

[17] Boykov, Yuri Y., and M-P. Jolly. "Interactive graph cuts for optimal boundary \& region segmentation of objects in ND images." In Proceedings eighth IEEE international conference on computer vision. ICCV 2001, vol. 1, pp. 105-112. IEEE, 2001.

[18]Lombaert, Herve, Yiyong Sun, Leo Grady, and Chenyang Xu. "A multilevel banded graph cuts method for fast image segmentation." In Tenth IEEE International Conference on Computer Vision (ICCV'05) Volume 1, vol. 1, pp. 259-265. IEEE, 2005.

[19] Gelbaum, Zach, Mathew Titus, and James Watson. "Multi-Scale Analysis on Complex Networks using Hermitian Graph Wavelets." arXiv preprint arXiv:1901.07051 (2019).

[20]Deng, Feiyue, Shaopu Yang, Yongqiang Liu, Yingying Liao, and Bin Ren. "Fault diagnosis of rolling bearing using the hermitian wavelet analysis, KPCA and SVM." In 2017 International Conference on Sensing, Diagnostics, Prognostics, and Control (SDPC), pp. 632-637. IEEE, 2017.

[21] Xue, Xiaofeng, Xuefeng Chen, Xingwu Zhang, and Baijie Qiao. "Hermitian plane wavelet finite element method: Wave propagation and load identification." Computers \& Mathematics with Applications 72, no. 12 (2016): 2920-2942.

[22] Chen, Jian, Wen Li, Qingdong Li, Peng Li, Chengbin Lian, and Zhang Ren. "Signal singularity detection based on the Hermitian wavelet for fault diagnosis." In 2014 International Conference on Cloud Computing and Big Data, pp. 116-118. IEEE, 2014.

[23] Li, Hui. "Multi-Scale Hermitian Wavelet Order Envelope Spectrum Based Bearing Fault Detection and Diagnosis." International Journal of Digital Content Technology and its Applications 7, no. 1 (2013): 440.

[24] Li, Hui, Yuping Zhang, and Haiqi Zheng. "Application of Hermitian wavelet to crack fault detection in gearbox." Mechanical Systems and Signal Processing 25, no. 4 (2011): 1353-1363.

[25]Peachap, Atemangoh Bruno, and Daniel Tchiotsop. "Epileptic seizures detection based on some new Laguerre polynomial wavelets, artificial neural networks and support vector machines." Informatics in Medicine Unlocked 16 (2019): 100209.

[26] Wang, Shuihua, Ming Yang, Yin Zhang, Jianwu Li, Ling Zou, Siyuan Lu, Bin Liu, Jiquan Yang, and Yudong Zhang. "Detection of left-sided and right-sided hearing loss via fractional Fourier transform." Entropy 18, no. 5 (2016): 194. 